\title{
INVENTORY ROUTING OF INDUSTRIAL GASES WITH STOCHASTIC DEMAND
}

\author{
by \\ Bhupeshkumar Gandhi \\ B.Eng.(Industrial Engineering) \\ Suarshtra University, \\ Gujarat, India, June 1991 \\ A project \\ presented to Ryerson University \\ in partial fulfillment of the \\ requirements for the degree of \\ Master of Engineering \\ in the Program of \\ Mechanical Engineering
}

Toronto, Ontario, Canada, 2010

(C) Bhupeshkumar Gandhi 2010 


\section{Author's Declaration}

I hereby declare that I am the sole author of this project.

I authorize Ryerson University to lend this project to other institutions or individuals for the purpose of scholarly research.

I further authorize Ryerson University to reproduce this project by photocopying or by other means, in total or in part, at the request of other institutions or individuals for the purpose of scholarly research. 


\section{Borrower's Page}

Ryerson University requires the signatures of all persons using or photocopying this project. Please sign below, and give address and date. 


\title{
INVENTORY ROUTING OF INDUSTRIAL GASES WITH STOCHASTIC DEMAND
}

\author{
Bhupeshkumar Gandhi \\ Master of Engineering, Mechanical Engineering, 2010 \\ Ryerson University
}

\begin{abstract}
This report proposes a methodology to solve the inventory routing problem of industrial gases with stochastic demand. The gas tanker distributes gases from a depot to several dispersed customers in a route, and each customer has stochastic demand modeled by a Brownian motion. The proposed model determines the optimal quantity required to refill each customer by minimizing the cost associated with earliness, which increases number of visits per year, and lateness, which increases probability of stockout. Overall, the proposed model minimizes the total system cost, helps find the optimal tanker capacity for a given route, and improves supplier and customers' relationship. Numerical examples and sensitivity analysis are given to illustrate the proposed model.
\end{abstract}




\section{Acknowledgements}

I am deeply indebted to my project supervisor, Dr. Mohamed Wahab Mohamed Ismail, for his valued and generous guidance and encouragement. His patience has played fundamental role in the completion of this project. I am very grateful for giving me this opportunity to work under his supervision.

At the same time I appreciate the generous support given to me by my wife Sheetal Gandhi and my kids Vatsa and Nandani. 


\section{Table of Contents}

Author's Declaration $\quad$ ii

Borrower's Page $\quad$ iii

Abstract $\quad$ iv

Acknowledgement $\quad$ ' v

Table of Contents $\quad$ vi

List of Tables $\quad$ vii

List of Figures $\quad$ viii

Nomenclature $\quad$ x

1 Introduction $\quad 1$

2 Literature Review $\quad 4$

$\begin{array}{lll}3 & \text { The Model } & 7\end{array}$

3.1 Problem definition $\ldots \ldots \ldots \ldots \ldots$

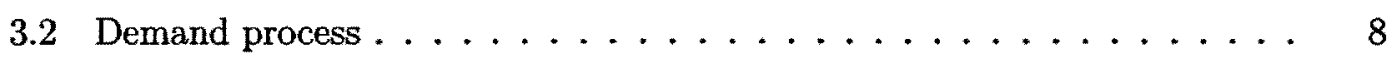

3.3 Model development . . . . . . . . . . . . . . . . . . . 10

3.3.1 The tanker visitation ........................ 12 
3.3 .2 Cost description . . . . . . . . . . . . . 13

3.3 .3 Overage cost $\ldots \ldots \ldots \ldots \ldots \ldots \ldots \ldots$

$3.3 .4 \quad$ Underage cost $\ldots \ldots \ldots \ldots \ldots \ldots \ldots \ldots$

3.3.5 The total cost $\ldots \ldots \ldots \ldots \ldots \ldots \ldots \ldots \ldots \ldots \ldots$

4 Examples and Sensitivity Analysis $\quad 25$

4.1 An example ......................... 25

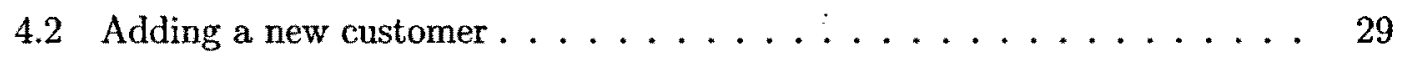

4.3 Changing safety stock level $\ldots \ldots \ldots \ldots \ldots \ldots \ldots \ldots \ldots$

4.4 Effect of overage cost and underage cost $\ldots \ldots \ldots \ldots \ldots$

5 Conclusion $\quad 35$

$\begin{array}{lr}\text { References } & 36\end{array}$ 


\section{List of Tables}

4.1 Numerical values for associated parameters . . . . . . . . . . 26

4.2 The total cost for different tank levels for Customer $1 \ldots \ldots \ldots 27$

4.3 The total cost for different tank levels for Customer $2 \ldots \ldots 27$

4.4 The total cost for different tank levels for Customer $3 \ldots \ldots \ldots 27$

4.5 The total cost for each customer . . . . . . . . . . . 30

4.6 The optimal quantity and the total cost for Customer 4 - case I . . . 30

4.7 The optimal quantity and the total cost for Customer 4 - case II . . . . 31

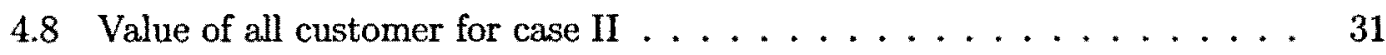

4.9 At different safety levels, the tank level and the total cost for each customer 32

4.10 Total cost and cycle time for overage cost and underage cost $\ldots \ldots .34$ 


\section{List of Figures}

3.1 Demand probability density function (pdf) with Time . . . . . . . 9

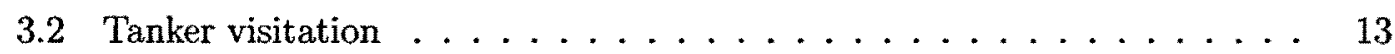

3.3 Cost-tank level structure from (Berman and Larson 2001) . . . . . . 15

3.4 Expected inventory level in a customer's tank . . . . . . . . . 16

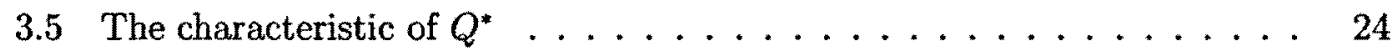

4.1 The total cost with respect to the quantity for each customer . . . . . . 28

4.2 Effect of the mean and variance of demand on the total cost $\ldots \ldots 32$

4.3 The tank level and the total cost for different safety levels . . . . . . 33

4.4 Effect of overage cost and underage cost on total cost for Customer 1 . . 34 


\section{Nomenclature}

$\mu \quad$ Brownian motion drift parameter (mean demand per unit time)

$\sigma^{2} \quad$ Variance of demand per unit time

$C_{o i} \quad$ Overage cost for customer $i$

$C_{u i} \quad$ Underage cost for customer $i$

$D(t) \quad$ Cumulative stochastic demand from time 0 to $t$

$\widehat{Q_{i}} \quad$ Approximate tank level for customer $i$

$Q_{i}^{*} \quad$ Optimal tank level for individual visit to customer $i$

$Q_{i} \quad$ Adjusted optimal tank level for customer $i$ based on cycle time

$C\left[Q_{i}, D_{i}(t)\right]$ Total cost for customer $i$

$k_{i} \quad$ Tank capacity for customer $i$

$s_{i} \quad$ Ideal refilling point for customer $i$ (in \% of the tank capacity)

C Tanker capacity

$d_{0 i} \quad$ Distance between depot and the customer $i$

$d_{i j} \quad$ Distance between customer $i$ to customer $j$

$r \quad$ Instantaneous discount rate

$T \quad$ Cycle time for the route

$T_{i} \quad$ Cycle time for individual visit to customer $i$ from the depot

$T(Q) \quad$ First passage time when the cumulative demand first exceeds the level $Q$

$u(t ; Q) d t \quad$ The probability that exactly $t$ time units have elapsed at the time when demand first exceeds the level $Q$

$v(z ; t) d z \quad$ The probability that demand will change by exactly $z$ units, given that $t$ units of time have elapsed

$w(z ; t, Q) \quad$ The probability that the cumulative demand will be $z$ at time $t$, without previously exceeding the level $Q$ 


\section{Chapter 1}

\section{Introduction}

This study focuses on industrial gases distribution motivated by two main considerations: (i) importance of logistics and distribution system in the world economy; and (ii) current trend in supply chain coordination.

Industrial gases (e.g., Nitrogen, Oxygen, Acetylene, Argon) used in a variety of industries such as chemicals and refining-related processes, metal manufacturing and fabrication, electronics, medical, food, paper and pulp, glass, and mining. The estimated demand of industrial gases grows about $5 \%$ to $6 \%$ annually worldwide. The worldwide demand was $\$ 31$ billion in 2009 and forecasted to be about $\$ 54$ billion in 2014 (Freedonia 2010). Industrial gases are manufactured in a highly automated plant located at one place and supplied to the customers at disperse locations. These products are distributed by using different modes to meet the demand of a wide range of industries. The main distribution modes are pipelines, tanker trailers, and cylinders.

Logistics and distribution systems have significant impact on the current economy. In United States, the logistics cost in 2007 was $\$ 1.4$ trillion, just above $10 \%$ of the nominal gross domestic product (GDP)(LogisticsToday 2008). Surprisingly, 48\% (\$671 billion) of this logistics cost is spent on the mode of tanker trailers; and $79 \%$ ( $\$ 487$ billion) of this cost is transportation and inventory carrying costs. Considering an individual product, 
manufacturing cost is fairly uniform among different suppliers. However, transportation and holding cost, between $10 \%$ and $30 \%$ of a product's sales price (Ghiani et al. 2004), is the key factor in the total cost. As the demand forecast grows about $5 \%$ annually, an efficient distribution system is required to stay competitive.

The second aspect of the industrial gases distribution involves in the current trend of supply chain coordination. An inventory routing problem consists of two different supply chain problems: inventory management and vehicle routing. In inventory management, the main objective is to determine the optimal order quantity minimizing the total cost of the inventory system. While in vehicle routing problem, the main objective is to minimize the total transportation cost from a depot to dispersed customers. The coordination of inventory and transportation is only possible when customers are part of the system and the customers value can be created through timely delivery, product availability, and consistency of product. Therefore, the main objective of the inventory routing problem is to minimize inventory cost and transportation cost while ensuring the product availability. This is a win-win situation to improve the relationship between supplier and customers.

The inventory routing problem has been studied by a number of authors since 1970 . Different authors focus on different characteristics to solve inventory routing problems for different products. Bell et al. (1983) are among the first to study the industrial gases distribution problem assuming deterministic demand, finite discrete time, and variable static routes. Golden et al. (1984) consider probabilistic demand to minimize the total cost using a heuristic method to select the customer to be visited. Berman and Larson (2001) also study the inventory routing of industrial gases distribution assuming probabilistic demand and determine the optimal required quantity for each customer in a given route. While Berman and Larson (2001) introduce the industrial gases distribution problem with a stochastic demand describing by a Brownian motion to represent the realworld problem, in the subsequent modeling approach, which is a dynamic programming, 
they consider the probabilistic demand for each customer. In short, the existing studies model the inventory routing problem considering either deterministic or probabilistic demand and uses heuristic or dynamic programming approach to solve the problem.

The main difference between the proposed model and the existing models in the literature is that the customer demand follows a stochastic process modeled by a Brownian motion with drift, $\mu$, and variance, $\sigma^{2}$, per unit time. The proposed model considers a safety stock level for each customer and determines the optimal quantity to refill each customer in a route by minimizing earliness cost (overage cost) and lateness cost (underage cost).

The remainder of this report is organized as follows: Chapter 2 provides a brief introduction of inventory routing problem and literature review. Chapter 3 presents and solves the inventory routing of industrial gases with stochastic demand modeled by a Brownian motion. Chapter 4 illustrates several numerical examples by using the proposed model in Chapter 3. In addition, Chapter 4 presents sensitivity analysis related to safety stock, overage and underage costs, and adding new customer in a route. 


\section{Chapter 2}

\section{Literature Review}

This chapter provides a brief introduction about the basic concept of inventory routing problem and includes a briefing of the problem as analyzed by multiple authors.

Inventory routing problem is a core problem of supply chain that is solved by using Vendor Managed Inventory (VMI). VMI has numerous benefits over traditional inventory management and vehicle routing. The traditional inventory management system constitutes the customers monitoring their own inventory levels and determining time for reorder. In vehicle routing problems a supplier has to supply according to customers order and time. In vehicle routing the customer has to keep more inventories to avoid stockout resulting in increased inventory holding cost. The supplier has to maintain individual delivery time for each customer, increasing the distribution cost. Thus, as there is no proper coordination between the supplier and customer the total cost increases. In contrast to this, in VMI the supplier monitors the customers' inventory level and delivery time avoiding stockout. This allows lower inventory holding cost for retailer (Day et al. 2009). In this process the supplier coordinates delivery quantity and route for each customer thereby reducing the overall transportation cost and stockout scenarios (Zhao et al. 2008). This results in the increased service level for the end user and the customer. By using VMI as stated the customer does not need extra resources to monitor their in- 
ventory. The benefits of VMI has been successful realized by Wal-Mart and key suppliers like Proctor and Gamble, Fruit of the Loom (Cetinkaya and Lee 2000).

The inventory routing problem covers two classical problems of supply chain, inventory control and vehicle routing. A firm with minimal resources provides distribution of a product from its central distribution warehouse to geographically dispersed customers. These customers have limited storage capacity and stochastic demands. This concept leads to the most common benefit of lower inventory carrying cost, lower transportation cost and minimizing chances of shortage or stockout (Adelman 2004). Example of industries using this process are petrochemical industries, air-product industries, chemical industries, beverage distribution and cash replenishment to automatic teller machines (ATMs). This problem has been studied by a number of authors since 1970's for different products and a few of them discussed in this section.

Bell et al. (1983) are among the first to work on inventory control with vehicle scheduling for industrial gases distribution. For vehicle scheduling problem, the decision support system was used that emphasized on scheduling a vehicle as per the customer order so as to minimize mileage and at the same time take care that the customer does not fall short of the product.

Federgruen and Zipkin (1984) assume random customer demand with non-linear cost function and limited depot capacity. Cost function covers inventory holding costs, transportation costs, and shortage costs for each customer. The objective of this model is to determine delivery routes that minimize the total cost incurred during single day. The procedure exchanges customers among routes iteratively.

Golden et al. (1984) also consider a single day large scale inventory problem and minimize inventory and transportation costs. Golden et al. (1984) use a heuristic method to calculate urgency of each customer and compare it with the set target value. This urgency calculates the ratio of tank inventory level to tank size. Dror and Ball (1987) consider deterministic daily consumption rate to calculate replenishment time for each 
customer. It considers inventory routing on short term planning period to reduce risk of stockout.

Anily and Federgruen (1990) study long term replenishment strategies to minimize system wide inventory and transportation cost. This model considers constant deterministic demand to minimize long-range average inventory and transportation costs. Webb and Larson (1995) discuss strategic inventory routing problem related to Larson (1988). It focuses on a distribution system to minimize fleet șize for servicing customers.

Day et al. (2009) define a heuristic model to minimize transportation and refilling time. This model focuses on routing of the vehicle to cover all customers without experiencing stockout while considering deterministic demand.

In this project, the proposed model is closely related to Berman and Larson (2001) discussing an inventory routing problem for industrial gases. Berman and Larson (2001) indicate that the customer demand follows a stochastic process - a Brownian motion. However, in model formulation and numerical examples, Berman and Larson (2001) assume that the demand is represented by a truncated Gaussian distribution. The quantity delivered to the customer depends on the emptiness of customers' tank or amount available on board and customers to be visited. It considers an incremental cost structure depending on earliness or lateness of delivery and the return of the tanker to depot empty or non-empty. 


\section{Chapter 3}

\section{The Model}

This chapter presents a model for inventory routing of industrial gases with stochastic demand modeled by a Brownian Motion. This model considers a single depot, a single vehicle, a single product, and multiple customers. The depot needs to route a large industrial tanker to minimize earliness and lateness costs of each customer so that the optimal quantity required to refill each customer, the cycle time of the route, and the tanker capacity for a given route can be determined. It is assumed that a route can be completed by visiting every customer in a route within the cycle time. As per the above assumption, the minimum mean first passage time is equal to the cycle time, which is the sum of the service time for each customer, time to visit the first customer from the depot, and completing the route visiting all customers from one to another. This implies that the route and the total distance travelled by the tanker visiting each customer and returning back to depot are fixed. Therefore, in this model, in optimization, the cost related to the distance is not considered.

\subsection{Problem definition}

Let's consider a two-level supply chain distribution system with a centrally located depot and geographically dispersed $n$ customers. The depot has unlimited supply quantity of 
an industrial gases. An industrial gases tanker has limited capacity, $C$, and customer $i$ has tank capacity, $k_{i}$, and safety level, $s_{i}$, which is considered as the ideal refilling point, where $i=1,2,3, \ldots, n$. The tanker leaves the depot to deliver quantity $\sum_{i=1}^{n}\left(Q_{i}-s_{i}\right)$, where $Q_{i}\left(\leq k_{i}\right)$ is the tank level for customer $i$, to $n$ customers in the given route and then return to the depot. Each customer has stochastic demand $D_{i}(t)$, where $\left\{D_{i}(t), t \geq 0\right\}$ and is a Brownian motion with drift, $\mu_{i}$, and variance, $\sigma_{i}^{2}$, per unit time. The objective is to find the refilling quantity, $\left(Q_{i}-s_{i}\right)$, for customer $i$, and cycle time, $T$, for a given route, so that when the tanker visits customer $i$ its inventory level is at the ideal safety level, $s_{i}$.

\subsection{Demand process}

In the literature, it is often assumed that the customer demand for industrial gases is deterministic (e.g., Bell et al. 1983, Anily and Federgruen 1990, Savelsbergh and Song 2007) and probabilistic (e.g.,Golden et al. 1984). However, Berman and Larson (2001) indicate that the customer demand for industrial gases follows a Brownian motion process in real-world application. A Brownian motion process is defined by Karlin and Taylor (1975)(page 356) as follows:

A Brownian motion or Wiener process with drift is a stochastic process $\{D(t) ; t \geq 0\}$ with the following properties:

(a) Each increment $D\left(t_{2}\right)-D\left(t_{1}\right)$ is normally distributed with mean $\mu\left(t_{2}-t_{1}\right)$ and variance $\sigma^{2}\left(t_{2}-t_{1}\right)$, with $\mu, \sigma$ being fixed constants;

(b) For every pair of disjoint time intervals $\left[t_{1}, t_{2}\right],\left[t_{3}, t_{4}\right]$, say, $t_{1}<t_{2}<t_{3}<t_{4}$, the increments $D\left(t_{4}\right)-D\left(t_{3}\right)$ and $D\left(t_{2}\right)-D\left(t_{l}\right)$ are independent random variables with distributions given in (a), and similarly for $n$ disjoint intervals, where $n$ is an arbitrary positive integer; 
(c) $D(0)=0$ and $D(t)$ is continuous at $t=0$.

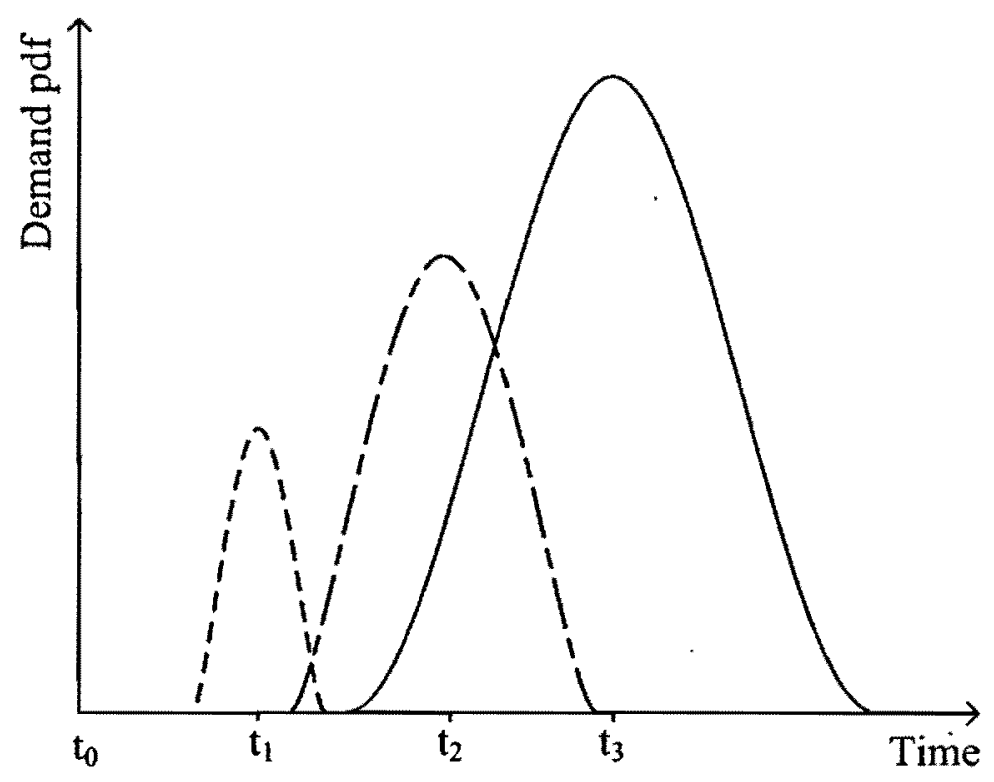

Figure 3.1: Demand probability density function (pdf) with Time

Figure 3.1 represents the relationship between time and demand probability density function (pdf). At $t_{0}=0$ demand $D(0)=0$ and variance $\sigma^{2}=0$. As the time increases from $t_{0}<t_{1}<t_{2}<t_{3}$, the mean demand rate and variance of demand increases (Berman and Larson 2001). This does not mean that the demand can go to infinity with a period. Based on the above explanation, the customer's demand of industrial gases at time $t_{0}=0$ is $D(0)=0$, where $t_{0}$ is the time just after the customer tank is refilled up to its optimal tank level $Q^{*}$. In the next visit, the amount of industrial gases that can be filled in the customer's tank is either $Q^{*}$, if the tank is completely empty, or the available capacity of the tank up to $Q^{*}$ is also a normal random variable with a Brownian motion. 


\subsection{Model development}

In order to define the stochastic demand process modeled by the Brownian motion, our approach relies on works done by Feller (1957), Manne (1961), and Chao (1992). In stochastic demand process, a random variable $T(Q)$, which is the first passage time when cumulative demand first exceeds the tank level $Q$, is defined by Karlin and Taylor (1975) (page 361),

$$
T(Q)=\inf \{t \mid D(t) \geq \dot{Q}\}
$$

To begin with our model, the first step is to derive an expression for the mean first passage time of the Brownian motion that is defined by Karlin and Taylor (1975)(page $362)$ as follows:

$$
\mathbb{E}\left[e^{-r T}\right]=e^{\theta Q}
$$

where

$$
\theta=\frac{-\mu \pm \sqrt{\mu^{2}+2 \sigma^{2} r}}{\sigma^{2}}
$$

and $r$ denotes the instantaneous discount rate. This model considers $\theta \geq 0$. Now substituting value of

$$
\theta=\frac{-\mu+\sqrt{\mu^{2}+2 \sigma^{2} r}}{\sigma^{2}}
$$

in Equation (3.2), we get

$$
\mathbb{E}\left[e^{-r T}\right]=\exp \left[-\frac{Q}{\sigma^{2}}\left(\sqrt{\mu^{2}+2 \sigma^{2} r}-\mu\right)\right] .
$$

By differentiating Equation (3.4) with respect to $r$, we get the following:

$$
\mathbb{E}\left[e^{-r T}(-T)\right]=\exp \left[-\frac{Q}{\sigma^{2}}\left(\sqrt{\mu^{2}+2 \sigma^{2} r}-\mu\right)\right]\left[-\frac{Q}{\sigma^{2}}\left(\frac{1}{2} 2 \sigma^{2}\left(\mu^{2}+2 \sigma^{2} r\right)^{-\frac{1}{2}}\right)\right] .
$$

Substituting $r \rightarrow 0$, we get the mean first passage time as,

$$
\mathbb{E}[T]=\frac{Q}{\mu} .
$$

The next step is to derive the probability density function for cumulative demand that does not previously exceed the $\operatorname{tank}$ level $Q$ within time $t$. In order to achieve this, we consider the following definitions provided by Manne (1961) (page 646): 
$u(t ; Q) d t$ is the probability that exactly $t$ time units have elapsed at the time when demand first exceeds its initial level by $Q$ units (absorbing barrier at the origin, with the particle initially located $Q$ units above the origin).

$v(z ; t) d z$ is the probability that demand will change by exactly $z$ units, given that $t$ units of time have elapsed: a normal density function with mean $\mu t$ and variance $\sigma^{2} t$ (unconstrained random walk with the particle initially at the origin).

$w(z ; t, Q)$ represents the probability that the cumulative demand will be $z$ at time $t$, without previously exceeding its level $Q$.

The relationship of $u(t ; Q) d t, v(z ; t) d z$, and $w(z ; t, Q)$ is defined as:

$$
w(z ; t, Q)=v(z, t)-\int_{\tau=0}^{t} u(\tau, Q) v(z-Q, t-\tau) d z d \tau .
$$

The Laplace transforms of $u, v$, and $w$ are represented by $\bar{u}, \bar{v}$, and $\bar{w}$, respectively, and the Laplace transformation of Equation (3.6) is given as

$$
\bar{w}(z ; r, Q)=\bar{v}(z, r)-\bar{u}(r, Q) \bar{v}(z-Q, r)
$$

where

$$
\bar{u}(r, Q)=\int_{t=0}^{\infty} u(t, Q) e^{-r t} d t=e^{\lambda Q},
$$

and

$$
\bar{v}(z, r)= \begin{cases}\frac{\lambda \nu}{r(\lambda-\nu)} e^{\lambda z}, & \text { if } z \geq 0 \\ \frac{\lambda \nu}{r(\lambda-\nu)} e^{\nu z}, & \text { if } z<0\end{cases}
$$

where

$$
\lambda=\frac{\mu-\sqrt{\mu^{2}+2 \sigma^{2} r}}{\sigma^{2}}, \quad \nu=\frac{\mu+\sqrt{\mu^{2}+2 \sigma^{2} r}}{\sigma^{2}}, \quad \lambda<0, \quad \text { and } \quad \nu>0 .
$$

Substituting Equations (3.8) and Equation (3.9) in Equation (3.7), we get

$$
\bar{w}(z ; r, Q)= \begin{cases}\frac{\lambda \nu}{r(\lambda-\nu)}\left[e^{\lambda z}-e^{\lambda Q+\nu(z-Q)}\right], & \text { if } z \geq 0, \\ \frac{\lambda \nu}{r(\lambda-\nu)}\left[e^{\nu z}-e^{\lambda Q+\nu(z-Q)}\right], & \text { if } z<0 .\end{cases}
$$


In our model, we only require the case where $z \geq 0$ as the demand is always positive or zero. The probability density function in Equation (3.11) will be used to derive the expected costs in Sections (3.3.3) and (3.3.4). Before presenting the expected cost associated with our model, we will explain how the industrial gases tanker visitation process takes place in the next section.

\subsubsection{The tanker visitation}

Let's consider three customers: Customer 1, Customer 2, and Customer 3 located in a given route (see Figure 3.2). The distance between the depot and each customer is $d_{01}, d_{02}, d_{03}$, where, for example, $d_{01}$ is the distance between the depot and the Customer 1. If the tanker visits each customer individually as shown in Figure 3.2 (a), the total distance covered to visit a single customer is twice the distance between the depot and the customer. Hence, the total distance covered to visit all three customers is:

$$
D_{\text {ind }}=2\left(d_{01}+d_{02}+d_{03}\right) \text {. }
$$

If the tanker visits all three customers in a cyclic route as shown in Figure 3.2 (b), the total distance covered from depot to visit each customer in the route and return back to the depot is

$$
D_{c y c}=d_{01}+d_{12}+d_{23}+d_{30} .
$$

As indicated, in the cyclic route, the total distance is distributed among all customers. The distance covered and the related cost for individual customer in the cyclic route is less than that of individual visitation. Let the required time for individual visitation, described in Figure 3.2(a), to Customer 1, Customer 2, and Customer 3 be $T_{1}, T_{2}$, and $T_{3}$, respectively. The ideal value for $T_{i}$ is the time when the inventory level of customer $i$ is at his/her safety level $s_{i}$. In other words, $T_{i}$ is the mean first passage time of customer $i$ to reach level $s_{i}$; and that can be determined by using Equation (3.5). Consequently, the tanker will not have neither cost of earliness (overage) nor cost of lateness (underage) 


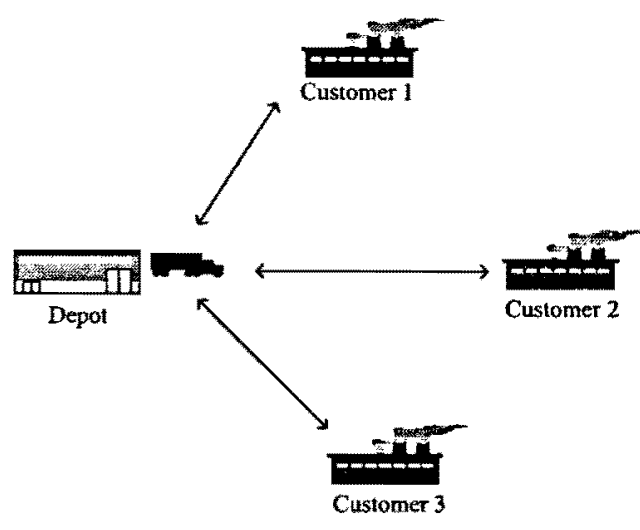

Individual visitation

(a)

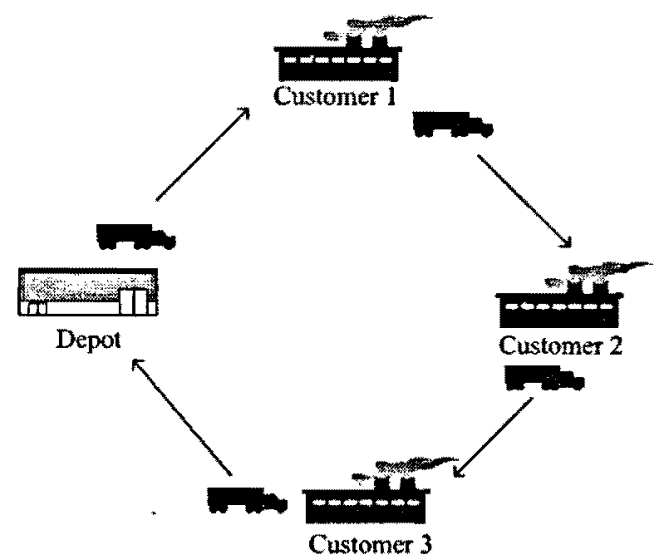

Cyclic route

(b)

Figure 3.2: Tanker visitation

as it always reaches the customer when the inventory level exactly at the safety level. Now, if these three customers are placed in a particular cyclic route as shown in Figure 3.2(b), the cycle time, $T$, for routing the tanker should satisfy the following relationship.

$$
T \leq \min \left[T_{1}, T_{2}, T_{3}\right]
$$

Otherwise, some of the customers' tank would be empty before the taker visits them for next refilling. Similarly, the routing time for $n$ customers can be generalized as follows:

$$
T \leq \min \left[T_{1}, T_{2}, T_{3}, \ldots, T_{n}\right]
$$

\subsubsection{Cost description}

The tanker visits each customer a number of times during a year. During each visit, a customer will have the cost of purchasing industrial gases and a charge for refilling based on the time and quantity refilled. In addition to these costs, there are some fixed costs related to the distance traveled and service provided, and these costs are discussed below:

The distance related charge depends on the total distance covered by the tanker and 
number of customers in the route. If the tanker visits more frequently to a customer this charge will increase; while if the tanker visits less frequently this charge will decrease. This charge is fixed per visit and does not depend on the refilling quantity.

The service charge is a time related charge and consists of the time required for safely parking the tanker at the customer's site, paper work for delivery, and gas hose connection and disconnection. These durations are fixed per visitation and do not depend on the refilling quantity. For example, a tanker delivers 500 liters of gases to the customer or 1000 liters of gases to the customer, the time required for parking, paper work, and gas hose connection and disconnection remains the same. Therefore, refilling a smaller amount of gases increases the unit cost compared to refilling a larger amount.

As the number of visits increases during a year, the total cost per year also increases. The number of visits per year depends on the tank refilling policy. If a customer's tank is refilled when it exactly reaches half of the tank level then the number of visits will be double compared to the case where the refilling takes place when the customer's tank is completely empty. Refilling a customer's tank when it is completely empty causes chances of stockout. As a result, early arrivals at a customer's site increase the number of visits per year and late arrivals increase chances of stockout. Therefore, it necessary to determine the optimal cycle time for a tanker to visit and the optimal refilling quantity for each customer in a route to minimize distance and service related costs per year.

Figure 3.3 represents the costs associated with the tank level. One can notice that every time tanker visits a customer when the inventory level is at $s$ there is no additional fixed charges. However, early arrival requiring an early visitation of the tanker at a customer's site causes less amount of quantity required to be filled in the customer's tank. Each early visitation to a customer increases some fractional visitations in the future. As discussed before, each visit incurs some fixed charges. Hence, increased number of fractional visitations increases the related fixed cost. Now, if the tanker arrives late when a customer's tank level is less than the ideal refilling point, $s$, it causes loss of goodwill 
in the eyes of the customer. If the tanker arrives when a customer's tank level is at 0 level, the customer suffers a complete stockout. Hence, late arrivals are associated with a huge penalty compared to earliness, as shown in Figure 3.3. Berman and Larson (2001) discuss these costs in detail and assume that the customer's ideal refilling point, $s$, is given.

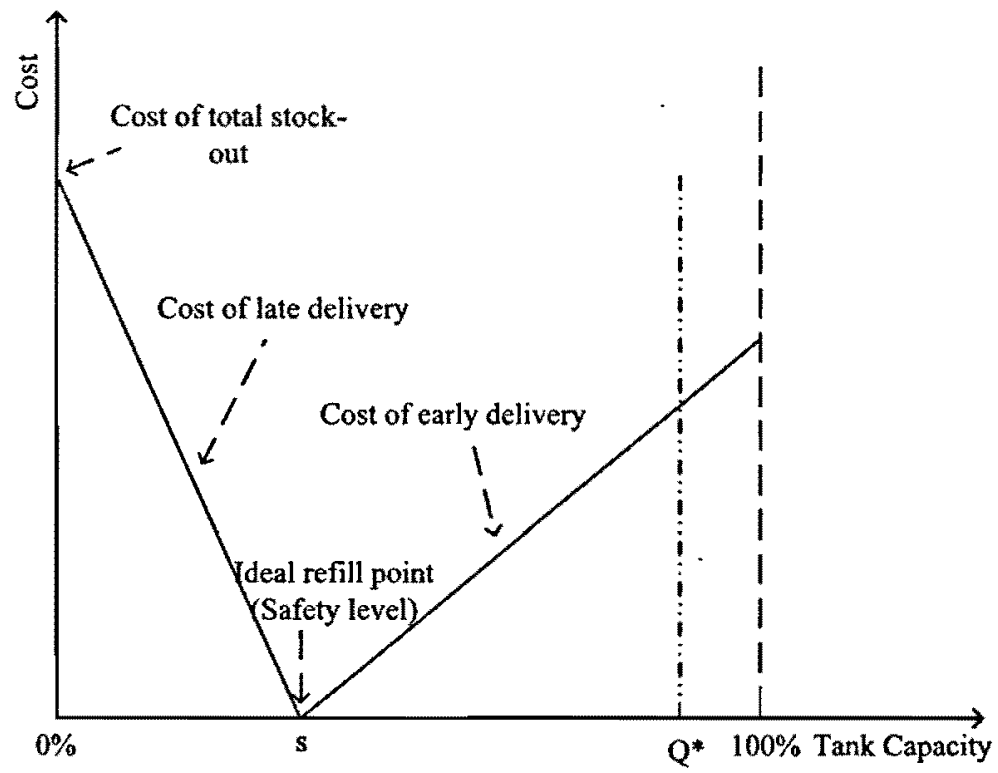

Figure 3.3: Cost-tank level structure from (Berman and Larson 2001)

Next we explain the cost structure based on the inventory level in a customer's tank. Figure 3.4 shows that at time $T(0)$, time just after refilling the customer's tank, the total inventory in the customer's tank is $Q$. The customer's tank will be empty in $T(Q)$ time unit. As discussed in Figure 3.3, $s$ is the ideal refilling point. Hence, the tanker needs to visit the customer when the inventory level is $s$. That means, the available inventory to satisfy the demand before the next refilling is $Q-s$ and it would take $T(Q-s)$ time unit. Now, during time $T(Q-s)$, if $D(t)<Q-s$ the tank level is above the ideal refilling point. This causes earliness cost (overage cost) that is defined as $C_{o}$ per unit. However, during time $T(Q-s)$, if $D(t)>Q-s$ the tank level is below the ideal refilling point. 
The cost is related to lateness (underage cost) and defined as $C_{u}$ per unit.

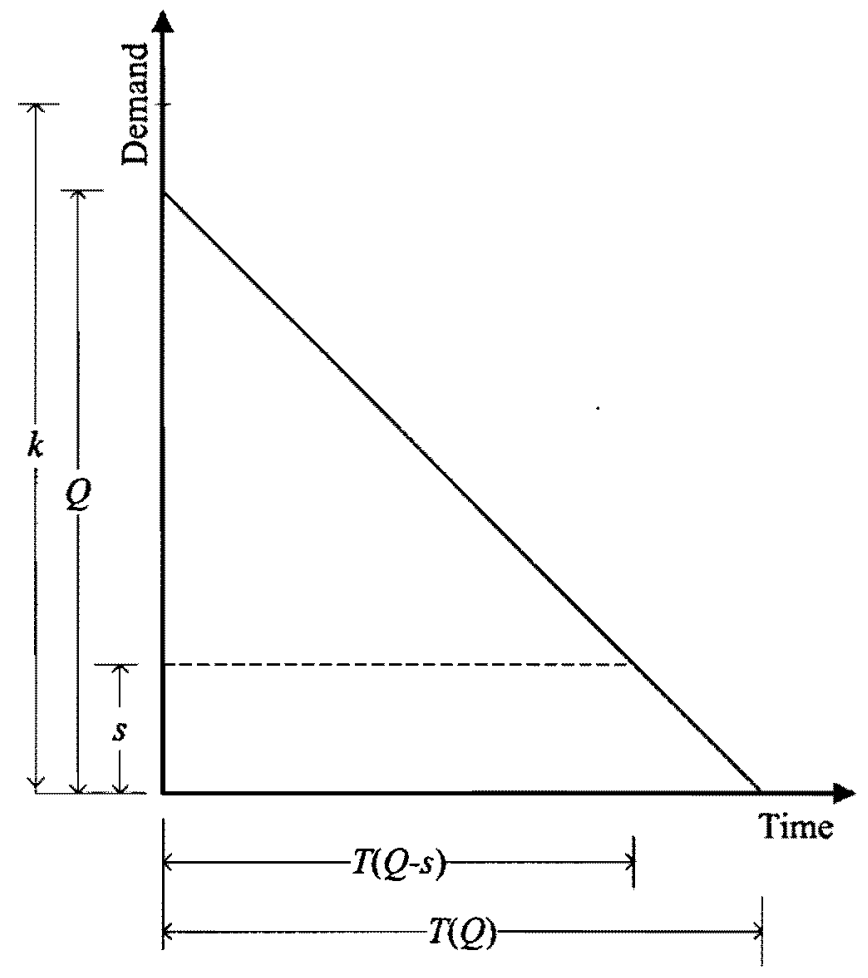

Figure 3.4: Expected inventory level in a customer's tank

\subsubsection{Overage cost}

The expected overage cost can be define as:

$$
\mathbb{E}\left[\int_{0}^{T(x)}[x-D(t)]^{+} d t\right],
$$

where $x$ is $Q-s$ and $[x-D(t)]^{+}=\max (x-D(t), 0)$. To determine the expectation given in Equation (3.13), first we derive the expected overage cost with the instantaneous discount rate $r$ and then substitute $r=0$. The expected overage cost with the instantaneous 
discount rate defined as:

$$
\mathbb{E}\left[\int_{0}^{T(x)} e^{-r t}[x-D(t)]^{+} d t\right]=\int_{-\infty}^{\infty} \int_{0}^{\infty} e^{-r t}(x-z) w(z ; t, x) d t d z
$$

where $z$ is the demand $D(t)$ and let Laplace transformation of $\int_{0}^{\infty} e^{-r t} w(z ; t, x) d t$ be $\bar{w}(z ; r, x)$ (see Equation (3.11)). Next, demand is non-negative and hence the lower limit for the demand $z$ is 0 . The available inventory before the next refilling is $Q-s=x$. In other words, the upper limit for demand $z$ is $x$. Substituting all, Equation (3.14) can be written as,

$$
=\int_{0}^{x}(x-z) \bar{w}(z ; r, x) d z
$$

Replacing the value of $\bar{w}(z ; r, x)$ from Equation $(3.11)$,

$$
\int_{0}^{x}(x-z) \bar{w}(z ; r, x) d z=\frac{\lambda \nu}{r(\lambda-\nu)} \int_{0}^{x}(x-z)\left(e^{z \lambda}-e^{x \lambda+(z-x) \nu}\right) d z .
$$

This can be simplified as

$$
=\frac{\lambda \nu}{r(\lambda-\nu)}\left[\frac{-1+e^{x \lambda}-x \lambda}{\lambda^{2}}-\frac{e^{x(\lambda-\nu)}\left(-1+e^{x \nu}-x \nu\right)}{\nu^{2}}\right] .
$$

Therefore, overage cost with an instantaneous discount rate is:

$$
\begin{aligned}
\mathbb{E}\left[\int_{0}^{T(x)} e^{-r t}[x-D(t)]^{+} d t\right] & =\frac{-\nu^{2}+\nu^{2} e^{x \lambda}-x \lambda \nu^{2}-\lambda^{2} e^{x \lambda}}{\lambda \nu r(\lambda-\nu)} \\
& +\frac{\lambda^{2} e^{x(\lambda-\nu)}+x \lambda^{2} \nu e^{x(\lambda-\nu)}}{\lambda \nu r(\lambda-\nu)}
\end{aligned}
$$

In order to simplify Equation (3.15), we consider the first part of Equation (3.15),

$$
\frac{-\nu^{2}+\nu^{2} e^{x \lambda}-x \lambda \nu^{2}-\lambda^{2} e^{x \lambda}}{\lambda \nu r(\lambda-\nu)}
$$


Expanding it by using Taylor's theorem up to the terms of second order, we have the following expression:

$$
\begin{aligned}
& =\frac{-\nu^{2}+\nu^{2}\left(1+x \lambda+\frac{x^{2} \lambda^{2}}{2}\right)-x \lambda \nu^{2}-\lambda^{2}\left(1+x \lambda+\frac{x^{2} \lambda^{2}}{2}\right)}{\lambda \nu r(\lambda-\nu)}, \\
& =\frac{-2 \nu^{2}+2 \nu^{2}+2 x \lambda \nu^{2}+x^{2} \lambda^{2} \nu^{2}-2 x \lambda \nu^{2}-2 \lambda^{2}-2 x \lambda^{3}-x^{2} \lambda^{4}}{2 \lambda \nu r(\lambda-\nu)}, \\
& =\frac{x^{2} \lambda \nu^{2}-2 \lambda-2 x \lambda^{2}-x^{2} \lambda^{3}}{2 \nu r(\lambda-\nu)} .
\end{aligned}
$$

Taking the limit $r \rightarrow 0$, which satisfies the condition $\frac{0}{0}$, and applying L.Hospital's rule while substituting values of $\lambda$ and $\nu$ from Equation (3.10) in the abolve expression, the first part of Equation (3.15) can be written as

$$
\frac{-\nu^{2}+\nu^{2} e^{\lambda x}-x \lambda \nu^{2}-\lambda^{2} e^{\lambda x}}{\lambda \nu r(\lambda-\nu)}=\frac{x^{2}}{2 \mu}-\frac{\sigma^{4}}{4 \mu^{3}} .
$$

Simililaly, simplifying second part of Equation (3.15) by using Taylor's theorem up to terms of second order, taking the limit $r \rightarrow 0$ which satisfies the condition $\frac{0}{0}$, and applying L.Hospital's rule while substituting values of $\lambda$ and $\nu$ from Equation (3.10), it can be written as follows:

$$
\frac{\lambda^{2} e^{(\lambda-\nu) x}+x \nu \lambda^{2} e^{(\lambda-\nu) x}}{\lambda \nu r(\lambda-\nu)}=\frac{\sigma^{4} e^{\frac{-2 \mu x}{\sigma^{2}}}}{4 \mu^{3}}+\frac{x \sigma^{2} e^{\frac{-2 \mu x}{\sigma^{2}}}}{2 \mu^{2}} .
$$

Now, substituting Equations (3.16) and (3.17), the overage cost given in Equation (3.13) can be expressed as:

$$
\mathbb{E}\left[\int_{0}^{T(x)}[x-D(t)]^{+} d t\right]=\frac{x^{2}}{2 \mu}+\frac{\sigma^{2} x e^{\frac{-2 \mu x}{\sigma^{2}}}}{2 \mu^{2}}-\frac{\sigma^{4}}{4 \mu^{3}}\left(1-e^{\frac{-2 \mu x}{a^{2}}}\right) .
$$

\subsubsection{Underage cost}

The expected underage cost can be defined as,

$$
\mathbb{E}\left[\int_{0}^{T(x)}[x-D(t)]^{-} d t\right]
$$


where $x$ is $Q-s$ and $[x-D(t)]^{-}=\max (D(t)-x, 0)$. To determine the value of the expectation given in (3.19), first we consider the expected underage cost function with a instantaneous discount rate $r$ and then substitute $r=0$. The expected underage cost with instantaneous discount rate defined as:

$$
\mathbb{E}\left[\int_{0}^{T(x)} e^{-r t}[x-D(t)]^{-} d t\right]=\int_{-\infty}^{\infty} \int_{0}^{\infty} e^{-r t}(x-z) w(z ; t, x) d t d z .
$$

If demand exceeds more than the available quantity $x$ it causes lateness. As a customer's tank capacity is finite, the maximum demand that can be satisfied by a customer is truncated to its tank capacity $k$. As a result, demand ranges from $x$ to the customer's tank capacity $k$ will cause the lateness; and hence Equation (3.20) is written as

$$
=\int_{x}^{k}(x-z) \bar{w}(z ; r, x) d z
$$

Substituting the value of $\bar{w}(z ; r, x)$ from Equation (3.11), this can be expressed as

$$
=\frac{\lambda \nu}{r(\lambda-\nu)} \int_{x}^{k}(x-z)\left(e^{\lambda z}-e^{\lambda x+\nu(z-x)}\right) d z .
$$

After integration, the above expression becomes

$$
=\frac{\lambda \nu}{r(\lambda-\nu)}\left[\frac{-e^{\lambda x}+e^{\lambda k}(1-\lambda k+\lambda x)}{\lambda^{2}}-\frac{e^{\lambda x}\left(-1+e^{\nu(k-x)}(1-\nu k+\nu x)\right.}{\nu^{2}}\right] .
$$

Therefore, the underage cost with instantaneous discount rate can be writen as

$$
\begin{aligned}
\mathbb{E}\left[\int_{0}^{T(x)} e^{-r t}[x-D(t)]^{-} d t\right]= & \frac{-\nu^{2} e^{\lambda x}+\nu^{2} e^{\lambda k}-k \lambda \nu^{2} e^{\lambda k}+x \lambda \nu^{2} e^{\lambda k}+\lambda^{2} e^{\lambda x}}{\lambda \nu r(\lambda-\nu)} \\
& +\frac{-\lambda^{2} e^{(\lambda-\nu) x+\nu k}+k \lambda^{2} \nu e^{(\lambda-\nu) x+\nu k}-x \lambda^{2} \nu e^{(\lambda-\nu) x+\nu k}}{\lambda \nu r(\lambda-\nu)}
\end{aligned}
$$

In order to simplify Equation (3.21), we consider the first part of Equation (3.21),

$$
\frac{-\nu^{2} e^{\lambda x}+\nu^{2} e^{\lambda k}-k \lambda \nu^{2} e^{\lambda k}+x \lambda \nu^{2} e^{\lambda k}+\lambda^{2} e^{\lambda x}}{\lambda \nu r(\lambda-\nu)}
$$


Expanding it by using Taylor's theorem up to terms of second order, it becomes

$$
\begin{aligned}
= & \frac{-\nu^{2}\left(1+\lambda x+\frac{\lambda^{2} x^{2}}{2}\right)+\nu^{2}\left(1+\lambda k+\frac{\lambda^{2} k^{2}}{2}\right)-k \lambda \nu^{2}\left(1+\lambda k+\frac{\lambda^{2} k^{2}}{2}\right)}{\lambda \nu r(\lambda-\nu)} \\
& +\frac{x \lambda \nu^{2}\left(1+\lambda k+\frac{\lambda^{2} k^{2}}{2}\right)+\lambda^{2}\left(1+\lambda x+\frac{\lambda^{2} x^{2}}{2}\right)}{\lambda \nu r(\lambda-\nu)}, \\
= & \frac{1}{2 \lambda \nu r(\lambda-\nu)}\left[-2 \nu^{2}-2 x \lambda \nu^{2}-x^{2} \lambda^{2} \nu^{2}+2 \nu^{2}+2 k \lambda \nu^{2}+k^{2} \lambda^{2} \nu^{2}-2 k \lambda \nu^{2}\right. \\
& \left.-2 k^{2} \lambda^{2} \nu^{2}-k^{3} \lambda^{3} \nu^{2}+2 x \lambda \nu^{2}+2 x k \lambda^{2} \nu^{2}+x k^{2} \lambda^{3} \nu^{2}+2 \lambda^{2}+2 x \lambda^{3}+x^{2} \lambda^{4}\right], \\
= & \frac{-x^{2} \lambda \nu^{2}-k^{2} \lambda \nu^{2}-k^{3} \lambda^{2} \nu^{2}+2 x k \lambda \nu^{2}+x k^{2} \lambda^{2} \nu^{2}+2 \lambda+2 x \lambda^{2}+x^{2} \lambda^{3}}{2 \nu r(\lambda-\nu)} .
\end{aligned}
$$

Taking the limit $r \rightarrow 0$, which satisfies the condition $\frac{0}{0}$, and applying L.Hospital's rule while inserting values of $\lambda \nu$ from Equation (3.10), first part of Equation (3.21) can be expressed as

$$
\frac{-\nu^{2} e^{\lambda x}+\nu^{2} e^{\lambda k}-k \lambda \nu^{2} e^{\lambda k}+x \lambda \nu^{2} e^{\lambda k}+\lambda^{2} e^{\lambda x}}{\lambda \nu r(\lambda-\nu)}=\frac{-(k-x)^{2}}{2 \mu}+\frac{\sigma^{4}}{4 \mu^{3}} .
$$

Similarly, simplifying the second part of Equation (3.21) by using Taylor's theorem up to terms of second order, taking the limit $r \rightarrow 0$, which satisfies the condition $\frac{0}{0}$, and applying L.Hospital's rule while inserting values of $\lambda \nu$ from Equation (3.10), it can be expressed as

$$
\begin{aligned}
\frac{-\lambda^{2} e^{(\lambda-\nu) x+\nu k}+\lambda^{2} k \nu e^{(\lambda-\nu) x+\nu k}-\lambda^{2} e \nu e^{(\lambda-\nu) x+\nu k}}{\lambda \nu r(\lambda-\nu)} & =\frac{-\sigma^{4} e^{\frac{-2 \mu(x-k)}{\sigma^{2}}}}{4 \mu^{3}} \\
& +\frac{\sigma^{2}(k-x) e^{\frac{-2 \mu(x-k)}{\sigma^{2}}}}{2 \mu^{2}}
\end{aligned}
$$

Substituting Equation (3.22) and (3.23) in Equation(3.19), the underage cost is written as

$$
\mathbb{E}\left[\int_{0}^{T(x)}[x-D(t)]^{-} d t\right]=-\frac{(k-x)^{2}}{2 \mu}+\frac{\sigma^{2}(k-x) e^{\frac{-2 \mu(x-k)}{\sigma^{2}}}}{2 \mu^{2}}+\frac{\sigma^{4}}{4 \mu^{3}}\left(1-e^{\frac{-2 \mu(x-k)}{\sigma^{2}}}\right) .
$$




\subsubsection{The total cost}

The total cost, $C[Q-s, D(t)]$, is the sum of overage cost and underage cost and can be defined as:

$$
C[Q-s, D(t)]=C_{o}[\operatorname{Max}\{Q-s-D(t), 0\}]+C_{u}[\operatorname{Max}\{D(t)-Q+s, 0\}]
$$

Thus, the expected total cost is expressed as,

$$
\begin{aligned}
G(Q-s) & =\mathbb{E}[C(Q-s), D(t)] \\
& =\mathbb{E}\left[C_{o} \operatorname{Max}\{Q-s-D(t), 0\}+C_{u} \operatorname{Max}\{D(t)-Q+s, 0\}\right], \\
& =C_{o} \mathbb{E}[\operatorname{Max}\{Q-s-D(t), 0\}]+C_{u} \mathbb{E}[\operatorname{Max}\{D(t)-Q+s, 0\}], \\
& =C_{o} \int_{0}^{T(Q-s)}[Q-s-D(t)]^{+} d t+C_{u} \int_{0}^{T(Q-s)}[Q-s-D(t)]^{-} d t .
\end{aligned}
$$

In Equation (3.26), the first part of the right hand side is the overage cost and the second part is the underage cost. Subtituting the values of overage and underage costs from Equations (3.18) and (3.24), respectively, in Equation (3.26), the total cost can be expressed as

$$
\begin{aligned}
& G(Q-s)=C_{o}\left[\frac{(Q-s)^{2}}{2 \mu}+\frac{\sigma^{2}(Q-s) e^{\frac{-2 \mu(Q-s)}{\sigma^{2}}}}{2 \mu^{2}}-\frac{\sigma^{4}}{4 \mu^{3}}\left(1-e^{\frac{-2 \mu(Q-s)}{\sigma^{2}}}\right)\right] \\
& +C_{u}\left[-\frac{(k-Q+s)^{2}}{2 \mu}+\frac{\sigma^{2}(k-Q+s) e^{\frac{-2 \mu(Q-s-k)}{\sigma^{2}}}}{2 \mu^{2}}+\frac{\sigma^{4}}{4 \mu^{3}}\left(1-e^{\frac{-2 \mu(Q-s-k)}{\sigma^{2}}}\right)\right]
\end{aligned}
$$

Minimum total cost occurs when the first order condition is satisfied. That is,

$$
\frac{d}{d Q}[G(Q-s)]=0
$$

and $G(Q-s)$ needs to be convex and that must satisfy

$$
\frac{d^{2}}{d Q^{2}}[G(Q-s)]>0, \quad \forall Q-s>0
$$

In Equation (3.27), only $Q$ is a variable. Hence, differentiating $G(Q-s)$ with respect 
to $Q$, we obtain

$$
\begin{aligned}
& \frac{d}{d Q}[G(Q-s)] \\
& =\frac{d}{d Q}\left\{C_{o}\left[\frac{(Q-s)^{2}}{2 \mu}+\frac{\sigma^{2}(Q-s) e^{\frac{-2 \mu(Q-s)}{\sigma^{2}}}}{2 \mu^{2}}-\frac{\sigma^{4}}{4 \mu^{3}}\left(1-e^{\frac{-2 \mu(Q-s)}{\sigma^{2}}}\right)\right]\right\} \\
& +\frac{d}{d Q}\left\{C_{u}\left[-\frac{(k-Q+s)^{2}}{2 \mu}+\frac{\sigma^{2}(k-Q+s) e^{\frac{-2 \mu(Q-s-k)}{\sigma^{2}}}}{2 \mu^{2}}+\frac{\sigma^{4}}{4 \mu^{3}}\left(1-e^{\frac{-2 \mu(Q-s-k)}{\sigma^{2}}}\right)\right]\right\} \\
& =C_{o}\left[\frac{(Q-s)}{\mu}-\frac{(Q-s) e^{\frac{-2 \mu(Q-s)}{\sigma^{2}}}}{\mu}+\frac{\sigma^{2} e^{\frac{-2 \mu(Q-s)}{\sigma^{2}}}}{2 \mu^{2}}-\frac{\sigma^{2} e^{\frac{-2 \mu(Q-s)}{\sigma^{2}}}}{2 \mu^{2}}\right] \\
& +C_{u}\left[\frac{k-Q+s}{\mu}-\frac{(k-Q+s) e^{\frac{-2 \mu(Q-s-k)}{\sigma^{2}}}}{\mu}-\frac{\sigma^{2} e^{\frac{-2 \mu(Q-s-k)}{\sigma^{2}}}}{2 \mu^{2}}+\frac{\sigma^{2} e^{\frac{-2 \mu(Q-s-k)}{\sigma^{2}}}}{2 \mu^{2}}\right] \text {, } \\
& =C_{o}\left[\frac{(Q-s)}{\mu}-\frac{(Q-s) e^{\frac{-2 \mu(Q-s)}{\sigma^{2}}}}{\mu}\right] \\
& +C_{u}\left[\frac{k-Q+s}{\mu}-\frac{(k-Q+s) e^{\frac{-2 \mu(Q-s-k)}{\sigma^{2}}}}{\mu}\right]
\end{aligned}
$$

Setting the resultant expression to zero yields the optimality condition. That is, $\frac{d}{d Q}[G(Q-s)]=0$

$$
\begin{aligned}
& C_{o}\left[\frac{(Q-s)}{\mu}-\frac{(Q-s) e^{\frac{-2 \mu(Q-s)}{\sigma^{2}}}}{\mu}\right] \\
& +C_{u}\left[\frac{k-Q+s}{\mu}-\frac{(k-Q+s) e^{\frac{-2 \mu(Q-s-k)}{\sigma^{2}}}}{\mu}\right]=0 .
\end{aligned}
$$

In order to simplify above expression, considering Taylor's theorem up to terms of first order, we obtain the following:

$C_{o}\left[(Q-s)\left\{1-\left(1-\frac{2 \mu(Q-s)}{\sigma^{2}}\right)\right\}\right]+C_{u}\left[(k-Q+s)\left\{1-\left(1-\frac{2 \mu(Q-s-k)}{\sigma^{2}}\right)\right\}\right]=0$.

After simplifying we get,

$$
C_{o}(Q-s)^{2}=C_{u}(k-Q+s)^{2}
$$

Now, taking positive square $\operatorname{root}^{1}$ on both side,

$$
(Q-s) \sqrt{C_{o}}=(k-Q+s) \sqrt{C_{u}}
$$

\footnotetext{
${ }^{1}$ Negative square root gives $Q=k \frac{\sqrt{C}}{\sqrt{C_{u}}-\sqrt{C_{o}}}+s>k$ is not feasible given that $C_{u}>C_{o}$
} 
Therefore, approximate optimum quantity,

$$
\widehat{Q}=\frac{(k+s) \sqrt{C_{u}}+s \sqrt{C_{o}}}{\sqrt{C_{o}}+\sqrt{C_{u}}}
$$

Since we consider Taylor's theorem up to terms of first order, quantity, $\widehat{Q}$, obtained in equation (3.30) is an approximation. As a result, the refilling quantity $\widehat{Q}-s$ is also an approximation.

The optimal $Q^{*}$ must satisfy that $\frac{d}{d Q}[G(Q-s)]=0$. Thus, defining $\frac{d}{d Q}[G(Q-s)]=$ $f^{\prime}(Q-s)=0$, The $Q^{*}$ must satisfy $f^{\prime}\left(Q^{*}-s\right)=0$, which is Equation (3.29). One can find $Q^{*}$ using an iterative method considering $\widehat{Q}$ as the initial value.

\section{The property of the optimal quantity}

Rewriting Equation (3.29), we obtain the following

$$
\begin{aligned}
f^{\prime}(Q-s) & =C_{o}\left[\frac{(Q-s)}{\mu}-\frac{(Q-s) e^{\frac{-2 \mu(Q-s)}{\sigma^{2}}}}{\mu}\right]+C_{u}\left[\frac{k-Q+s}{\mu}-\frac{(k-Q+s) e^{\frac{-2 \mu(Q-s-k)}{\sigma^{2}}}}{\mu}\right] \\
& =\frac{C_{o}(Q-s)}{\mu}\left[1-\frac{1}{e^{\frac{2 \mu(Q-s)}{\sigma^{2}}}}\right]-\frac{C_{u}(k-Q+s)}{\mu}\left[-1+e^{\frac{2 \mu(k-Q+s)}{\sigma^{2}}}\right]
\end{aligned}
$$

The right hand side of Equation (3.31), let us consider the first term, where $C_{o}>0, Q>s$ and hence $1 / e^{\frac{2 \mu(Q-s)}{\sigma^{2}}}<1$. Therefore, the first term is always positive and it is linearly increases in $Q$. Regarding the second term, $C_{u}>0, k>Q$, and hence $e^{\frac{2 \mu(k-Q+s)}{\sigma^{2}}}>0$. Therefore, the second term is always positive with a negative sign and it is exponentially increases in $Q$. One can also notice that the first term is much smaller than the second term. Consequently, the value of $f^{\prime}(Q-s)<0, \forall Q<Q^{*}$. These characteristics are presented in Figure 3.5. In Equation (3.31), in order to find $Q^{*}$, in each iteration, one should increase value of $Q$ from the initial value of $\widehat{Q}$ until $f^{\prime}(Q-s)=0$.

In order prove the convexity of the total cost, differentiating Equation (3.28), we 


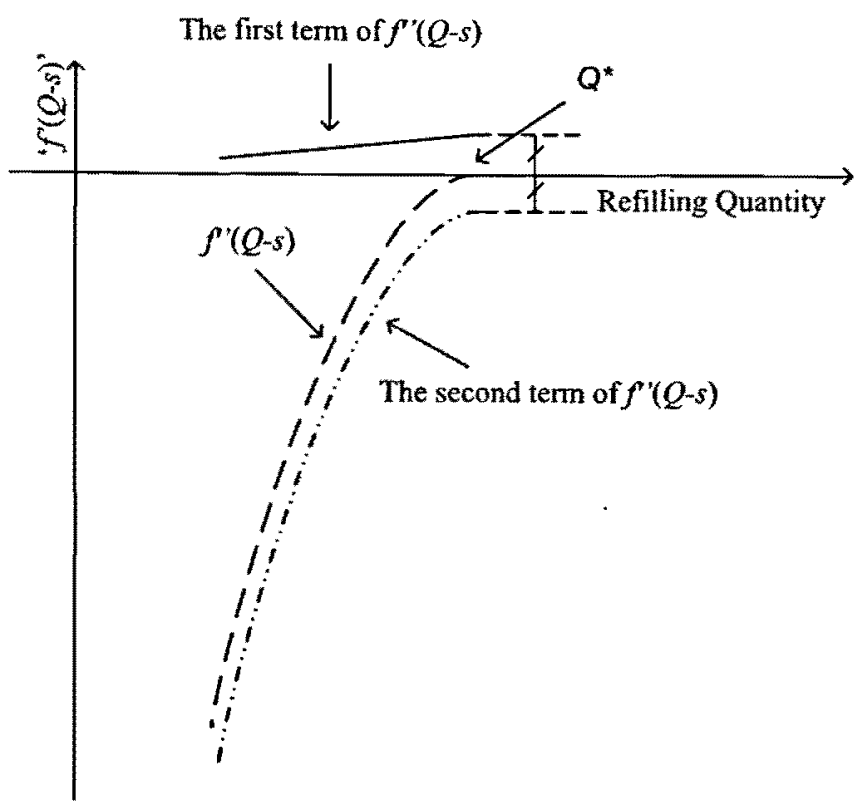

Figure 3.5: The characteristic of $Q^{*}$

obtain

$$
\begin{aligned}
\frac{d^{2}}{d Q^{2}}[G(Q-s)]= & \frac{d}{d Q}\left\{C_{o}\left[\frac{(Q-s)}{\mu}-\frac{(Q-s) e^{\frac{-2 \mu(Q-s)}{\sigma^{2}}}}{\mu}\right]\right\} \\
& +\frac{d}{d Q}\left\{C_{u}\left[\frac{k-Q+s}{\mu}-\frac{(k-Q+s) e^{\frac{-2 \mu(Q-s-k)}{\sigma^{2}}}}{\mu}\right]\right\} \\
& =C_{o}\left[\frac{1}{\mu}-\frac{e^{\frac{-2 \mu(Q-s)}{\sigma^{2}}}}{\mu}+\frac{2(Q-s) e^{\frac{-2 \mu(Q-s)}{\sigma^{2}}}}{\sigma^{2}}\right] \\
& +C_{u}\left[-\frac{1}{\mu}+\frac{e^{\frac{-2 \mu(Q-s-k)}{\sigma^{2}}}}{\mu}+\frac{2(k-Q+s) e^{\frac{-2 \mu(Q-s-k)}{\sigma^{2}}}}{\sigma^{2}}\right] .
\end{aligned}
$$

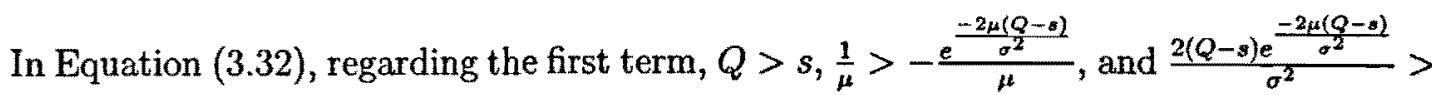
0 . As a result, so first term is always positive. With respect to the second term, $(k+s)>$ $Q, \frac{e^{\frac{-2 \mu(Q-s-k)}{\sigma^{2}}}}{\mu}>0, \frac{e^{\frac{-2 \mu(Q-s-k)}{\sigma^{2}-k}}}{\mu}>1 / \mu$, and $\frac{2(k-Q+s) e}{\sigma^{2}}{\frac{-2 \mu(Q-s-k)}{\sigma^{2}}}^{2}>0$. Therefore, the second term is always also positive. Consequently, $\frac{d^{2}}{d Q^{2}}[G(Q-s)]>0, \forall Q-s>0$, and the total cost function is convex. 


\section{Chapter 4}

\section{Examples and Sensitivity Analysis}

In this chapter, we explain our model presented in chapter 3 with numerical examples and sensitivity analysis related to safety stock, overage and underage costs, and adding new customer in a route.

\subsection{An example}

Consider a simple example with three customers $(n=3)$ and a depot as shown in Figure 3.2(b). The tanker leaves depot to deliver industrial gases to Customer 1. After that the tanker visits Customer 2 in the route, and finally visits Customer 3 and then return back to the depot. The ideal refilling point $s_{i}$ (for $i=1,2$, and 3 ) is $15 \%$ of the total tank capacity of each customer, the other related parameters are given Table 4.1.

In order to determine the optimum refilling quantity for each customer, first we use Equation (3.30) to find the approximate optimum quantity $\widehat{Q}_{\boldsymbol{i}}$.

Starting with Customer 1:

$$
\begin{aligned}
\widehat{Q}_{1} & =\frac{\left(k_{1}+s_{1}\right) \sqrt{C_{u 1}}+s_{1} \sqrt{C_{o 1}}}{\sqrt{C_{o 1}}+\sqrt{C_{u 1}}} \\
& =\frac{(1000+150) \sqrt{25}+150 \sqrt{15}}{\sqrt{15}+\sqrt{25}} \\
& =713.51 \text { ltrs. }
\end{aligned}
$$


Substituting $\widehat{Q}_{1}$ in Equation (3.31), we get,

$$
\begin{aligned}
& f^{\prime}\left(\widehat{Q}_{1}-s_{1}\right)=\frac{C_{o 1}\left(\widehat{Q}_{1}-s_{1}\right)}{\mu_{1}}\left[1-\frac{1}{e^{\frac{2 \mu_{1}\left(Q_{1}-s_{1}\right)}{\sigma_{1}^{2}}}}\right]-\frac{C_{u 1}\left(k_{1}-\widehat{Q}_{1}+s_{1}\right)}{\mu_{1}}\left[-1+e^{\frac{2 \mu_{1}\left(k_{1}-Q_{1}+s_{1}\right)}{\sigma^{2}}}\right] \\
& =\frac{15(713.51-150)}{330}\left[1-\frac{1}{e^{\frac{2 \times 330(713.51-150)}{87025}}}\right] \\
& -\frac{25(1000-713.51+150)}{330}\left[-1+e^{\frac{2 \times 330(1000-713.51+150)}{87025}}\right] \\
& =-847.55
\end{aligned}
$$

Table 4.1: Numerical values for associated parameters

\begin{tabular}{|l|c|c|c|}
\hline & Customer 1 & Customer 2 & Customer3 \\
\hline Tank capacity $\left(k_{i}\right)$ & 1000 & 1200 & 800 \\
Ideal refilling quantity $\left(s_{i}\right)$ & 150 & 180 & 120 \\
Overage cost $\left(C_{o i}\right)$ & 15 & 12 & 13 \\
Underage cost $\left(C_{u i}\right)$ & 25 & 20 & 22 \\
Mean demand per unit time $\left(\mu_{i}\right)$ & 330 & 410 & 260 \\
Variance per unit time $\left(\sigma_{i}^{2}\right)$ & 87025 & 122500 & 50625 \\
\hline
\end{tabular}

Now, increasing the value of $\widehat{Q}_{1}$, until it satisfies the condition $f^{\prime}\left(Q_{1}^{*}-s_{1}\right)=0$, we obtain $Q_{1}^{*}=974$ ltrs. Table 4.2 shows the values of $f^{\prime}\left(Q_{1}-s_{1}\right)$ for different values of $Q_{1}$. Similarly, we obtain $Q_{2}^{*}=1176$ for the customer 2 as shown in Table 4.3 , and $Q_{3}^{*}=787$ for the customer 3 as shown in Table 4.4. Figure 4.1 shows the convexity of the total cost for each customer with respect to the quantity.

Now, we calculate the mean first passage time for each customer by using Equation (3.5). The optimum quantity for Customer 1 is $Q_{1}=974$. The mean first passage time for Customer 1 is determined by

$$
\begin{aligned}
\mathbb{E}\left[T_{1}\right] & =\frac{Q_{1}^{*}-s_{1}}{\mu_{1}} \\
& =\frac{974-150}{330}=2.4970 \text { time unit. }
\end{aligned}
$$


Table 4.2: The total cost for different tank levels for Customer 1

\begin{tabular}{|c|c|c|}
\hline$Q_{1}$ & $f^{\prime}\left(Q_{1}-s_{1}\right)$ & the total cost, $G\left(Q_{1}-s_{1}\right)$ \\
\hline 713.51 & -847.55 & 83947.78 \\
800 & -321.11 & 36501.66 \\
900 & -73.21 & 18820.33 \\
950 & -17.62 & 16672.47 \\
974 & 0.06 & 16471.14 \\
990 & 9.45 & 16549.51 \\
1000 & 14.49 & 16669.71 \\
\hline
\end{tabular}

Table 4.3: The total cost for different tank levels for Customer 2

\begin{tabular}{|c|c|c|}
\hline$Q_{2}$ & $f^{\prime}\left(Q_{2}-s_{2}\right)$ & The total cost, $G\left(Q_{2}-s_{2}\right)$ \\
\hline 856.21 & -806.25 & 91386.52 \\
900 & -537.65 & 62315.31 \\
1000 & -193.47 & 28158.00 \\
1100 & -48.47 & 17121.56 \\
1150 & -12.75 & 15660.90 \\
1176 & 0.07 & 15503.27 \\
1200 & 9.31 & 15620.36 \\
\hline
\end{tabular}

Table 4.4: The total cost for different tank levels for Customer 3

\begin{tabular}{|c|c|c|}
\hline$Q_{3}$ & $f^{\prime}\left(Q_{3}-s_{3}\right)$ & The total cost, $G\left(Q_{3}-s_{3}\right)$ \\
\hline 572.31 & -994.47 & 73694.07 \\
600 & -673.68 & 50856.80 \\
700 & -130.81 & 16378.50 \\
750 & -36.63 & 12463.93 \\
787 & 0.45 & 11856.94 \\
800 & 9.29 & 11922.21 \\
\hline
\end{tabular}




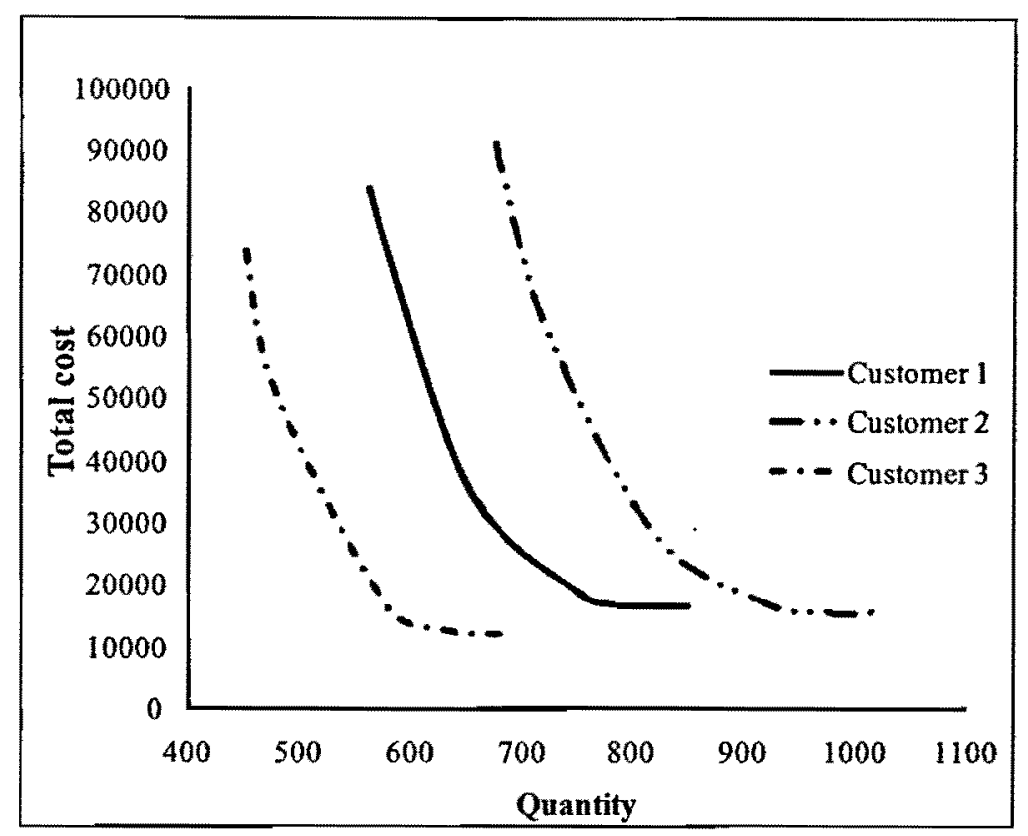

Figure 4.1: The total cost with respect to the quantity for each customer

Sirnilarly, the mean first passage time for Customer 2 is determined by

$$
\begin{aligned}
\mathbb{E}\left[T_{2}\right] & =\frac{Q_{2}^{*}-s_{2}}{\mu_{2}} \\
& =\frac{1176-180}{410}=2.4293 \text { time unit, }
\end{aligned}
$$

and the mean first passage time for customer 3 is determined by

$$
\begin{aligned}
\mathbb{E}\left[T_{3}\right] & =\frac{Q_{3}^{*}-s_{3}}{\mu_{3}} \\
& =\frac{787-120}{260}=2.5654 \text { time unit. }
\end{aligned}
$$

As we discussed in Chapter $3, \mathbb{E}\left[T_{i}\right]$ is the time to visit customer $i$ individually. Now the cycle time of the route must satisfy the condition defined in Equation (3.12):

$$
\begin{aligned}
T & =\min \left[T_{1}, T_{2}, T_{3}\right], \\
& =\min [2.4970,2.4293,2.5654], \\
& =2.4293 \text { time unit. }
\end{aligned}
$$


A cycle for each customer starts as the tanker leaves after refilling its tank. Since the tanker visits each customer every $T$ time instead of $T_{i}$, the refilling quantity changes accordingly. However, one of the customers refilling quantity will not change. In this particular example, refilling quantity of Customer 2 will not change, because it has the lowest mean first passage time and it should be one to be visited first in the route. It is assumed that after visiting Customer 2, the tanker first visits Customer 1 and then Customer 3. The refilling quantity for each customer is determined by using Equation (3.5).

$$
T=\frac{\left(Q_{i}-s_{i}\right)}{\mu_{i}} .
$$

The refilling quantity and refilling level for Customer 1, Customer 2 (unchanged) and Customer 3 are:

$$
\begin{aligned}
& Q_{1}-s_{1}=795, Q_{2}-s_{2}=996, \text { and } Q_{3}-s_{3}=625 \\
& Q_{1}=945, Q_{2}=1176, \text { and } Q_{3}=745 .
\end{aligned}
$$

The total tanker capacity is determined by $\sum_{i}^{n}\left(Q_{i}-s_{i}\right)$, where $n$ is the number of customers in the route. The required tanker capacity is $795+996+625=2416$. The total cost for each customer, as given in Table 4.5 , is determined by substituting these values of $k_{i}, Q_{i}, s_{i}, \mu_{i}$, and $\sigma_{i}^{2}$ in Equation (3.27) as follows:

$$
\begin{aligned}
& G\left(Q_{i}-s_{i}\right)=C_{o i}\left[\frac{\left(Q_{i}-s_{i}\right)^{2}}{2 \mu_{i}}+\frac{\left.\sigma_{i}^{2}\left(Q_{i}-s_{i}\right) e^{\frac{-2 \mu_{i}\left(Q_{i}-s_{i}\right)}{\sigma_{i}^{2}}}-\frac{\sigma_{i}^{4}}{4 \mu_{i}^{2}}\left(1-e^{\frac{\left.-2 \mu_{i} i Q_{i}-s_{i}\right)}{\sigma_{i}^{2}}}\right)\right]}{4 \mu_{i}^{3}}\right] \\
& +C_{u i}\left[-\frac{\left(k_{i}-Q_{i}+s_{i}\right)^{2}}{2 \mu_{i}}+\frac{\sigma_{i}^{2}\left(k_{i}-Q_{i}+s_{i}\right) e^{\frac{-2 \mu_{i}\left(Q_{i}-s_{i}-k_{i}\right)}{\sigma_{i}^{2}}}}{2 \mu_{i}^{2}}+\frac{\sigma_{i}^{4}}{4 \mu_{i}^{3}}\left(1-e^{\frac{-2 \mu_{i}\left(Q_{i}-s_{i}-k_{i}\right)}{\sigma_{i}^{2}}}\right)\right] .
\end{aligned}
$$

\subsection{Adding a new customer}

In this example, we add a new customer in the existing route and analyze the total cost and the refilling quantity. 
Table 4.5: The total cost for each customer

\begin{tabular}{|c|c|c|c|}
\hline & Customer 1 & Customer 2 & Customer 3 \\
\hline Total Cost & 16771.27 & 15503.27 & 12663.69 \\
\hline
\end{tabular}

\section{Case I:}

In previous example, there are three customers in the route, now we add one more customer the route with following characteristics:

$$
k_{4}=900, s_{4}=135, C_{04}=13, C_{u 4}=18, \mu_{4}=285, \sigma_{4}=57600 .
$$

The optimal refilling quantity, the total cost, and the mean first passage time for individual visit for Customer 4 are obtained and shown in Table 4.6. It can be seen that

Table 4.6: The optimal quantity and the total cost for Customer 4 - case I

\begin{tabular}{|c|c|c|}
\hline$Q^{*}$ & The total Cost & $\mathbb{E}\left[T_{4}\right]$ \\
\hline 883 & 13675.91 & 2.6246 \\
\hline
\end{tabular}

$\mathbb{E}\left[T_{4}\right]>T^{*}$, and therefore the cycle time for the route is unchanged $\left(T^{*}=2.4293\right)$. As a result, the total costs for all three Customers 1, 2, and 3 also remain the same. However, based on the cycle time, $T^{*}=2.4293$, the refilling quantity is adjusted and the total cost is determined for customer 4 as follows:

$$
Q_{4}=820, \text { and the total cost }=15530.17
$$

\section{Case II:}

Now, we add a new customer with higher mean demand rate and variance,

$$
\mu_{4}=350, \sigma_{4}=90000
$$

and keeping the other values the same. The optimal quantity, the total cost, and the mean first passage time for individual visit for customer 4 are obtained and shown in 
Table 4.7. It can be seen that $\mathbb{E}\left[T_{4}\right]<T^{*}$. Accordingly, the cycle time for the route is changed from $T^{*}=2.4293$ to $T^{*}=2.06857$.

Table 4.7: The optimal quantity and the total cost for Customer 4 - case II

\begin{tabular}{|c|c|c|}
\hline$Q^{*}$ & The total Cost & $\mathbb{E}\left[T_{4}\right]$ \\
\hline 859 & 10421.71 & 2.06857 \\
\hline
\end{tabular}

Since the cycle time reduces, refilling quantity and the total cost for other customers are also changed and shown in Table 4.8 .

Table 4.8: Value of all customer for case II

\begin{tabular}{|l|c|c|c|c|}
\hline & Customer 1 & Customer 2 & Customer 3 & Customer 4 \\
\hline Quantity $Q_{i}$ & 810 & 1006 & 640 & 859 \\
\hline The total cost & 33479.73 & 27035.79 & 30482.99 & 10421.71 \\
\hline
\end{tabular}

From Figure 4.2, we can see that as the mean rate and variance of demand of a new customer increase the cycle time of the route reduces. As a result, the refilling quantities reduce and the number of visits increases. This results in the increase of total cost for Customers 1,2,3 rapidly and the total cost for customer 4 decreases.

\subsection{Changing safety stock level}

In this section, we analyze the effect on quantity and the total cost for each customer by changing the safety stock level. In the first example, we consider safety stock level as $15 \%$ of the total tank capacity. By considering variable safety stock levels, the tank level and the total cost for each customer are determined and shown in Table 4.9.

Figure 4.3 indicates that as safety stock level increases from $0 \%$ to $20 \%$, the tank level, $Q_{i}$, increases but refilling quantity, $Q_{i}-s_{i}$, approximately remains the same and 


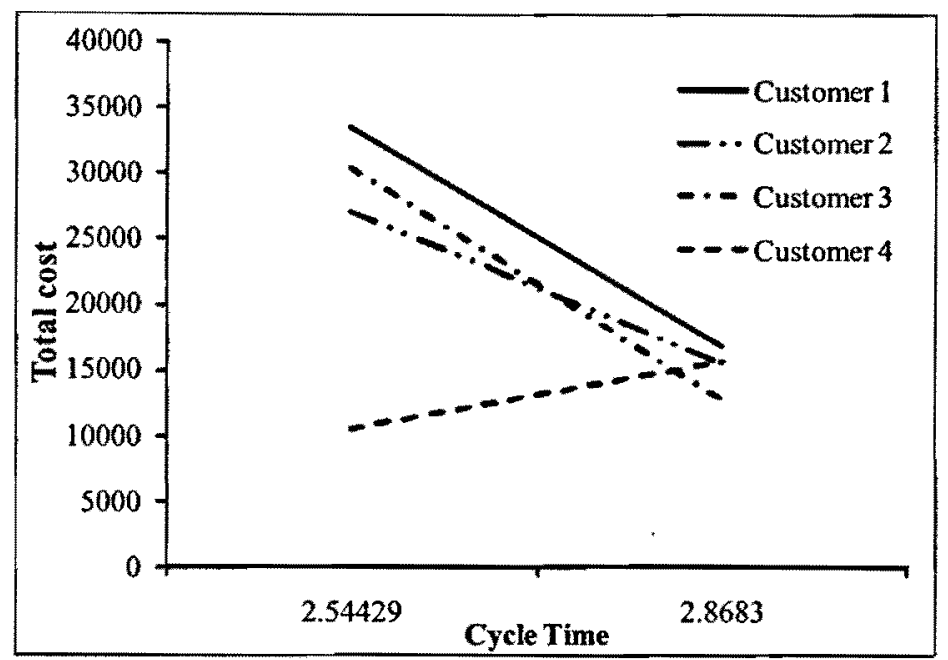

Figure 4.2: Effect of the mean and variance of demand on the total cost

Table 4.9: At different safety levels, the tank level and the total cost for each customer

\begin{tabular}{|c|c|c|c|c|c|}
\hline & $s$ & $5 \%$ & $10 \%$ & $15 \%$ & $20 \%$ \\
\hline \multirow{3}{*}{ Customer 1 } & The tank level & 850 & 900 & 945 & 1000 \\
\cline { 2 - 6 } & The total Cost & 16672.47 & 16672.47 & 16671.27 & 16672.47 \\
\hline \multirow{3}{*}{ Customer 2 } & The tank level & 1060 & 1115 & 1175 & 1200 \\
\cline { 2 - 6 } & The total Cost & 15507.00 & 15503.41 & 15503.27 & 15817.56 \\
\hline \multirow{2}{*}{ Customer 3 } & The tank level & 670 & 710 & 745 & 800 \\
\cline { 2 - 6 } & The total Cost & 12463.94 & 12463.94 & 12226.69 & 12169.56 \\
\hline
\end{tabular}

so the total cost also remain unchanged except at $s_{i}=20 \%$ where $Q=k$. At $s_{i}=20 \%$, Customer 1 refilling quantity $Q_{1}-s_{1}$ is unchanged (800) leaving the same cost. For Customer 2, refilling quantity $Q_{2}-s_{2}$ decreases from 1000 to 960 , because tank level $Q_{2}$ truncated at the tank capacity $k$, hence the total cost increases. While for Customer 3 , refilling quantity $Q_{3}-s_{3}$ increases from 630 to 640 , so the total cost decreases. 


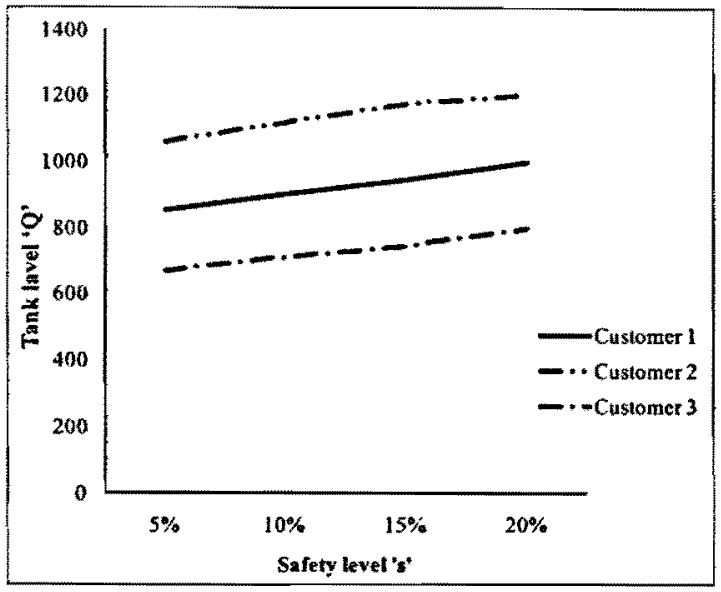

The tank level

(a)

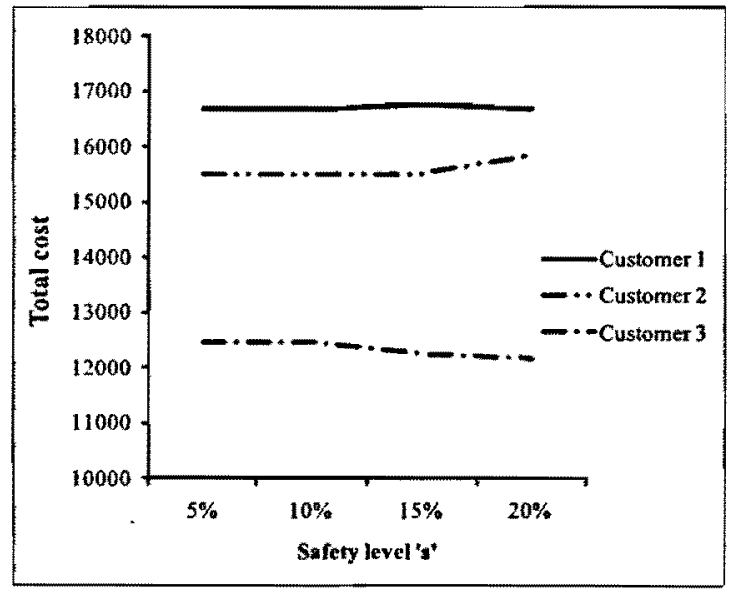

The total cost

(b)

Figure 4.3: The tank level and the total cost for different safety levels

\subsection{Effect of overage cost and underage cost}

In this section, we study the effect of overage cost and underage cost on the total cost for all customers in a route. In Table 4.10, we provide the total cost and cycle time for different overage and underage costs for Customer 1. It shows that for different overage and underage costs for customer 1 , the cycle time $\mathbb{E}\left[T_{1}\right]>T^{*}$, leaving the optimal refilling quantity unchanged.

Figure 4.4 shows that the total cost function linearly increases with increase of both costs, but overage cost has more impact on the total cost as compared to the underage cost. By changing any of these costs reduces the cycle time of the route, then it would increase the total cost of customers. 
Table 4.10: Total cost and cycle time for overage cost and underage cost

\begin{tabular}{|c|c|c|c|}
\hline$C_{o}$ & $C_{u}$ & The total cost for customer 1 & Cycle time \\
\hline 10 & 20 & 11583.37 & 2.98 \\
\hline 11 & 21 & 12616.65 & 2.98 \\
\hline 12 & 22 & 13649.93 & 2.97 \\
\hline 13 & 23 & 14683.21 & 2.96 \\
\hline 14 & 24 & 15716.50 & 2.96 \\
\hline 15 & 25 & 16749.78 & 2.95 \\
\hline 16 & 26 & 17658.00 & 2.95 \\
\hline 17 & 27 & 18816.34 & 2.94 \\
\hline 18 & 28 & 19849.62 & 2.94 \\
\hline 19 & 29 & 20882.90 & 2.94 \\
\hline 20 & 30 & 21916.18 & 2.93 \\
\hline
\end{tabular}

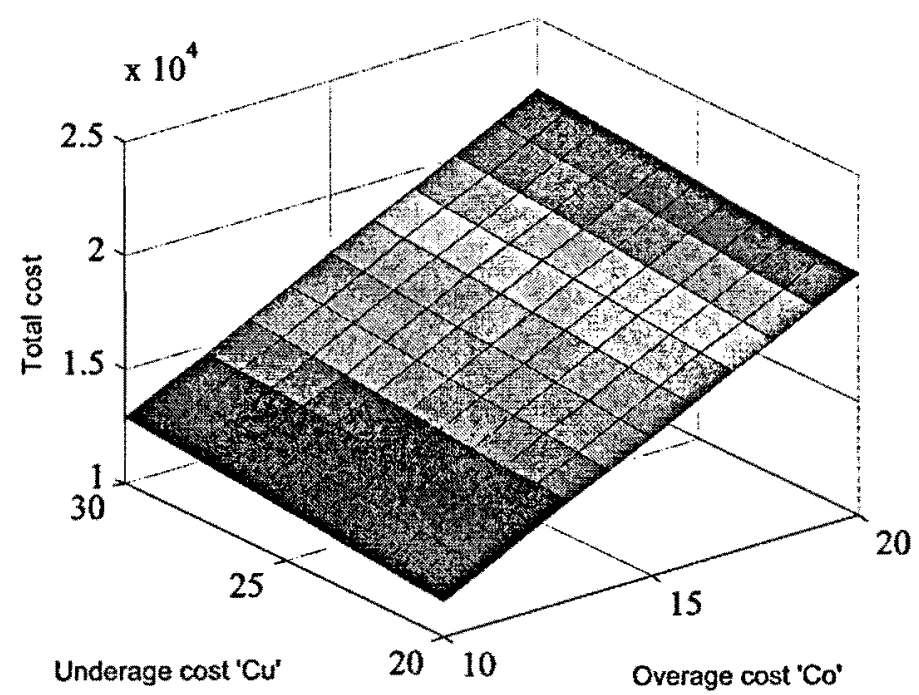

Figure 4.4: Effect of overage cost and underage cost on total cost for Customer 1 


\section{Chapter 5}

\section{Conclusion}

A mathematical model for inventory routing of industrial gases is developed to determine the amount of product required to refilling each customer in the route, the cycle time for the route, and the tanker capacity for the route. Unlike the existing literature, demand for each customer is considered to be stochastic and modeled by a Brownian motion. This model is useful to study the inventory routing problem with stochastic demand in a broad context beyond industrial gases. Numerical examples show that the higher the cycle time of the route the lower the total system cost. This is because when the cycle time increases, the number of visits decreases and the quantity to refill each customer increases. This will lead to less earliness (overage) and lateness (underage) costs and consequently the total system cost decreases. When the mean demand rate of a customer in the route increases, the cycle time reduces, i.e., the number of visits increases. As a result, the refilling quantity for the other customers in the route reduces. Earliness cost and lateness cost have also impact on the total system cost. It is assumed that a route can be completed by visiting every customers in a route within the cycle time. Further research can be directed to relax this assumption and also to incorporate the distance between customers. 


\section{References}

Adelman, D. (2004). A price-directed approach to stochastic inventory/routing. Operations Research, 52:499-514.

Anily, S. and Federgruen, A. (1990). One warehouse multiple retailer systems with vehicle routing costs. Management Science, 36:92-114.

Bell, W. J., Dalberto, L. M., Fisher, M. L., Greenfield, A. J., Jaikumar, R., Kedia, P., Mack, R. G., and Prutzman, P. J. (1983). Improving the distribution of industrial gases with an on-line computerized routing and scheduling optimizer. Interfaces, 6:4-23.

Berman, O. and Larson, R. (2001). Deliveries in an inventory/routing problem using stochastic dynamic programming. Transportation Science, 35:192-213.

Cetinkaya, S. and Lee, C.-Y. (2000). Stock replenishmenant shipment scheduling for vendor-managed inventory systems. Management Science, 46:217-232.

Chao, H.-P. (1992). The EOQ model with stochastic demand and discounting. European Journal of Operational Research, 59:434-443.

Day, J. M., Wright, P. D., Schoenherr, T., Venkataramanan, M., and Gaudette, K. (2009). Applications improving routing and scheduling decisions at a distributor of industrial gases. Omega, 37:227-237.

Dror, M. and Ball, M. (1987). Inventory routing: Reduction from an annual to a shortperiod problem. Naval Research Logistics, 34:891-905. 
Federgruen, A. and Zipkin, P. (1984). A combined vehicle routing and inventory allocation problem. Operations Research, 32:1019-1037.

Feller, W. (1957). An Introduction to Probability Theory and Its Applications, Vol.I, Second Edition. John Wiley \& Sons, Inc, New York.

Freedonia (2010). World industrial gases. http://www.freedoniagroup. com/brochure/ $26 \mathrm{xx} / 2659$ smwe.pdf.

Ghiani, G., Laporte, G., and Musmanno, R. (2004). Introduction to Logistics Systems Planning and Control. John Wiley \& Sons, Inc, New York.

Golden, B. L., Assad, A. A., and Dahl, R. (1984). Analysis of a large-scale vehicle routing problem with an inventory component. Large Scale Systems in Information and Decision Technologies, 7:181-190.

Karlin, S. and Taylor, H. M. (1975). A First Course in Stochestic Processes, Second Edition. Acadamic Press, New York.

Larson, R. C. (1988). Transporting sludge to the 106-mile site: An inventory/routing model for fleet sizing and logistic system design. Transportation Science, 22:186-198.

LogisticsToday (2008). US logistics cost top 10 percentage of GDP. http://ww. logisticstoday.com/operations_strategy/us_logistics_costs_0626\.

Manne, A. S. (1961). Capacity expansion and probabilistic growth. Econometrica, $29: 632-649$.

Savelsbergh, M. and Song, J.-H. (2007). Inventory routing with continuous moves. Computers $\mathcal{B}$ operations research, 34:1744-1763.

Webb, I. R. and Larson, R. C. (1995). Period and phase of customer replenishment: A new approach to the strategic inventory/routing problem. European Journal of Operational Research, 85:132-148. 
Zhao, Q.-H., Chen, S., and Zang, C.-X. (2008). Model and algorithm for inventory/routing decision in a three-echelon logistics system. European Journal of Operational Research, 191:623-635. 